\title{
Requirements for frameshift (deletion) during translesion synthesis
}

\author{
Shinya Shibutani * \\ Laboratory of Chemical Biology, Department of Pharmacological Sciences, State University of \\ New York at Stony Brook, Stony Brook, New York, 11794-8651, USA
}

\section{Summary}

Frameshift (deletion) is induced by many types of DNA damage in cells. However, the mechanism by which deletions are generated has not been extensively explored. The number of deletions during DNA synthesis catalyzed by the $3^{\prime} \rightarrow 5^{\prime}$ exonuclease-free $\left(\right.$ exo $\left.^{-}\right)$Klenow fragment of Escherichia coli DNA polymerase I (pol I) was determined systematically on dG-acetylaminofluorene (dG-AAF)-modified oligodeoxynucleotides templates with different bases 3 ' and/or 5' to the lesion. Under conditions where the dNMP (deoxynucleoside 3'-monophosphate) positions opposite dG-AAF can pair with its complementary base at the $5^{\prime}$ flanking position, one-base deletions are produced. Since the relative frequency of base insertion opposite the lesion followed the order: $\mathrm{dCMP}>\mathrm{dAMP}>\mathrm{dGMP}>\mathrm{dTMP}$, frequency of generating deletions paralleled to the insertion frequency of dNTP opposite the lesion. Inhibition of chain extension from the dC:dG-AAF pair also be involved in the formation of deletions. These results were supported by steady-state kinetic studies. Two and more base deletions were formed in a similar manner to that observed for one-base deletions. When the dG-AAF-modified templates containing iterated bases 5 ' to the lesion were used, shorter deletions predominated. The formation of deletions was reduced when exo ${ }^{+}$Klenow fragment was used, suggesting that the proofreading function of the enzyme minimizes the deletion formation. Thus, the ability of generating deletions depends on the $(a)$ the nature of base inserted opposite the lesion, $(b)$ sequence context to the lesion, and $(c)$ the overall rate of translesion DNA synthesis past the lesion. The mechanism for deletions by $E$. coli DNA pol I may be applied to predict the nature of deletions generated by a variety of DNA adducts and by other prokaryotic and eukaryotic DNA polymerases.

Keywords: frameshift, deletion, DNA adduct, translesion synthesis, DNA polymerase.

Base substitutions and frameshift (deletion) mutations generating in cells are associated with aging, genetic diseases and initiation of cancer. A base substitution induces a point mutation, resulting in a single incorrect amino acid in the protein sequence whereas a frameshift alters the entire amino acid sequence upstream of the mutation. Frameshift deletions are formed during translesion synthesis past endogenous or exogenous DNA damage (Ripley, 1990). Fresco and Alberts (1960) suggest-

\footnotetext{
*E-mail: shinya@pharm.sunysb.edu

Received: June 7, 2004, accepted: June 7, 2004

(C) Japanese Environmental Mutagen Society
}

ed that loops in duplex DNA might lead to the formation of deletions. The concept of slipped mispairing was proposed by Streisinger et al. (1966 and 1985) to account for the increased frequency of frameshift detected in iterated nucleotide sequences. Fowler et al. (1974) introduced a mechanism for substitution errors involving transient misalignment of the template-primer complex. Kunkel and co-workers (Kunkel and Soni, 1988; Bebenek and Kunkel, 1990; Kunkel, 1990) showed that frameshift deletions were induced by base mismatches, and proposed two alternative pathways; namely, misinsertion preceding misalignment (Fig. 1A) and misalignment pre-

This paper was presented to the symposium 2 "Mechanism of DNA damege and mutation" at the 32nd JEMS annual meeting, 2003. 
ceding misinsertion (Fig. 1B). I describe here describe the mechanism of frameshift (deletion) which occurs at DNA adducts during DNA synthesis catalyzed by DNA polymerases. Our results with damaged DNA adduct strongly support the former mechanism (Shibutani and Grollman, 1993; Shibutani et al., 2004).

\section{Capability of Formation of Deletions}

An in vitro experimental system has been used to demonstrate the formation of frameshift deletions and base substitutions occurring during DNA synthesis catalyzed by DNA polymerases (Shibutani, 1993; Shibutani and Grollman, 1993; Shibutani et al., 1996) . Primer extension reactions catalyzed by the $3 \rightarrow 5$ ' exonuclease-free $\left(\right.$ exo $\left.^{-}\right)$Klenow fragment of DNA polymerase I (pol I) were conducted on template oligodeoxynucleotides modified site-specifically with $N$-(deoxyguanosin-8-yl)-2-acety-

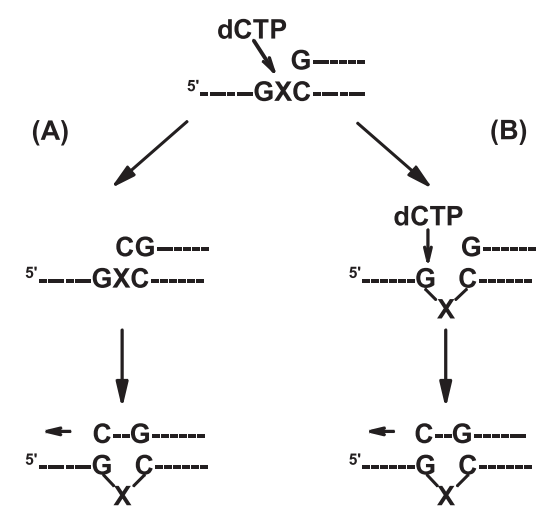

Fig. 1 A proposed frameshift (deletion) mechanism. (A) Misinsertion preceding misalignment. (B) Misalignment preceding misinsertion. laminofluorene (dG-AAF) in reaction mixture containing all four deoxynucleoside triphosphates (dNTPs) (Fig. 2) . The influence of DNA sequence context on the formation of deletions was determined by modifying bases 3 ' and/or 5 ' to the lesion systematically.

Using a ${ }^{32} \mathrm{P}$-labeled 13-mer primer with $\mathrm{dC}$ (13C) positioned opposite dG-AAF lesion, one-base deletion $\left(\Delta^{1}\right)$ was formed only when $\mathrm{G}$ is at the 5 ' flanking base (Fig. 3A). Similarly, dAMP, dGMP and dTMP were positioned opposite the lesion, one-base deletion was formed only when T, C and A, respectively, were at the 5' flanking base (Fig. 4A). Thus, one-base deletions are capable of forming under conditions where the dNMP positioned opposite dG-AAF can pair with the complementary base at the 5' flanking position.

The base located two positions 5' to the dG-AAF was varied systematically to establish the frequency of twobase deletion $\left(\Delta^{2}\right)$. Using a 13C-mer primer, two-base deletion was formed only when $\mathrm{G}$ is two bases 5 ' to the lesion (Fig. 3B). When 13A- and 13T-mer primers were used, two-base deletion was generated only when $\mathrm{T}$ and $\mathrm{A}$ were similarly positioned (Fig. 4B). Thus, two-base deletions are also capable of forming when the 5 ' flanking base can pair with the complementary base positioned opposite dG-AAF.

When a $13 \mathrm{G}$-mer primer is used, $80 \%$ one-base deletion and $15 \%$ two-base deletion were produced when $\mathrm{C}$ was two bases 5' to the lesion. A fraction of dGMP inserted opposite the dG-AAF lesion could pair with $\mathrm{C} 5$ ' to the lesion, resulting in the formation of one-base deletion (Fig. 5IA). The remaining fraction of dGMP inserted opposite the lesion and its 5' flanking G could pair with CC 5' to the lesion, forming two-base deletions (Fig. 5IB). In this case, the amount of two-base deletions was 5<smiles>C[C@@H](N(c1ccc2c(c1)Cc1ccccc1-2)c1nc2c(=O)[nH]c(N)nc2n1[R](F)(F)F)C(F)(F)F</smiles><smiles>Nc1nc2c(nc(Nc3ccc4c(c3)Cc3ccccc3-4)n2[R]2CCCCC2)c(=O)[nH]1</smiles>

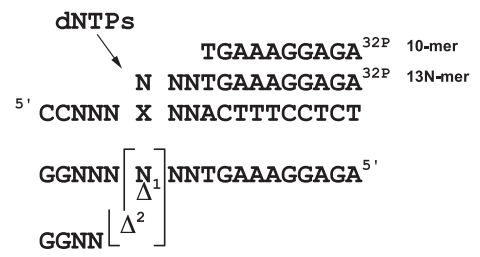

Fig. 2 Schematic diagram of the primer extension method. A dG-AAF-modified 18-mer template with different 3 ' and 5 ' bases to the lesion (5'CCNNNXNNACTTTCCTCT, X = dG-AAF or dG-AF; $\mathrm{N}=\mathrm{C}, \mathrm{A}, \mathrm{G}$ or T) was primed with a ${ }^{32} \mathrm{P}$-labeled 13 -mer or ${ }^{32} \mathrm{P}$-labeled 10 -mer. Primer extension reactions catalyzed by DNA polymerase were performed in the presence of four dNTPs. The extended products are compared with synthetic standards. 


$$
\mathbf{N}=\begin{array}{lllll}
\multicolumn{4}{c}{\text { CG-- }} \\
\multicolumn{1}{c}{\text { C }} & \text { A } & \text { G } & \text { T } & \text { Stn. }
\end{array}
$$

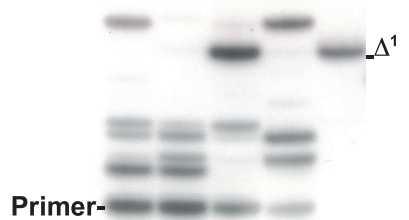

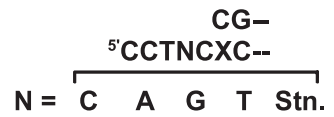

Primer-

Fig. 3 Effect of 5'-flanking base on the frequency of one-base or two-base deletions. (A) Using the dG-AAF-modified 18-mer template $\left({ }^{5}\right.$ CCTTNXCTACTTTCCTCT, $\mathrm{X}=\mathrm{dG}-\mathrm{AAF}, \mathrm{N}=\mathrm{C}, \mathrm{A}, \mathrm{G}$ or $\left.\mathrm{T}\right)$ primed with a ${ }^{32} \mathrm{P}$-labeled 13 -mer with $\mathrm{dC}$ at the 3 ' terminus (13Cmer), primer extension reactions were carried out in a buffer containing exo ${ }^{-}$Klenow fragment and four dNTPs. (B) Using the dG-AAF-modified 18-mer templates $\left({ }^{5}\right.$ CCTNCXCTACTTTCCTCT) primed with a ${ }^{32} \mathrm{P}$-labeled $13 \mathrm{C}$-mer, primer extension reactions were conducted, as described above. The reaction mixture was subjected to $20 \%$ polyacrylamide gel electrophoresis. Mobilities of reaction products were compared with a synthetic 17-mer containing a one-base deletion and 16-mer containing a two-base deletion.

A.
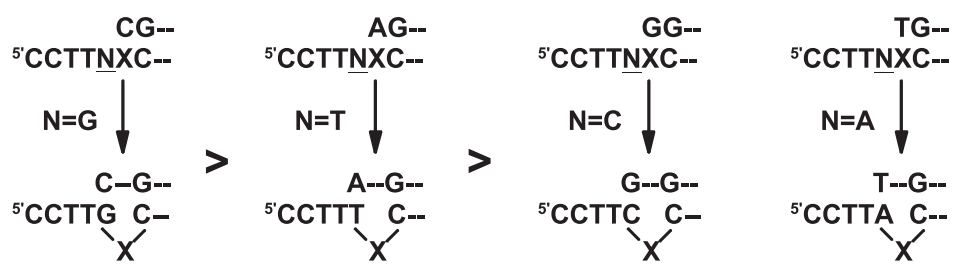

B.
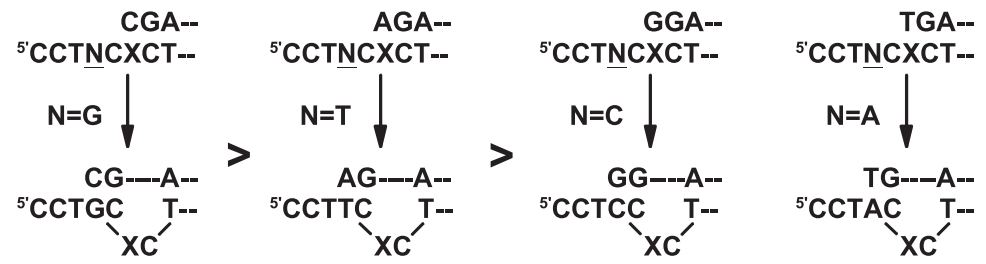

Fig. 4 A proposed mechanism for one-base and two-base deletions

fold lower than that of one-base deletions. Shorter deletions predominated.

Thus, deletions are capable of forming whenever the 3'terminus primer base (s), including dNMP inserted opposite the lesion, can pair correctly with the 5 ' neighboring base (s) to the lesion in template.

\section{Frequency of Nucleotide Insertion Opposite the dG-AAF Lesion}

In the previous sections, primers with dNMP positioned opposite the dG-AAF were used to explore the possible formation of one- and two-base deletions. To determine which dNMP is inserted preferentially opposite the lesion, kinetic analysis was performed to measure the relative frequency of base insertion $\left(F_{\text {ins }}\right)$ opposite dG-AAF (Shibutani and Grollman, 1993), according to a method developed by Mendelman et al. $(1989 ; 1990)$. The $F_{\text {ins }}$ for $\mathrm{dG}-\mathrm{AAF}$ was $\mathrm{dCMP}>\mathrm{dAMP}>\mathrm{dGMP}>\mathrm{dTTP}$ (Table 1). The frequency of base insertion opposite the lesion paralleled to the frequencies of one- and two-base deletions generated by dG-AAF (Shibutani and Grollman, 1993).

\section{Formation of Deletions during DNA Synthesis}

To determine the frequency of one- and two-base deletions formed during translesion synthesis past dG-AAF, 
I.
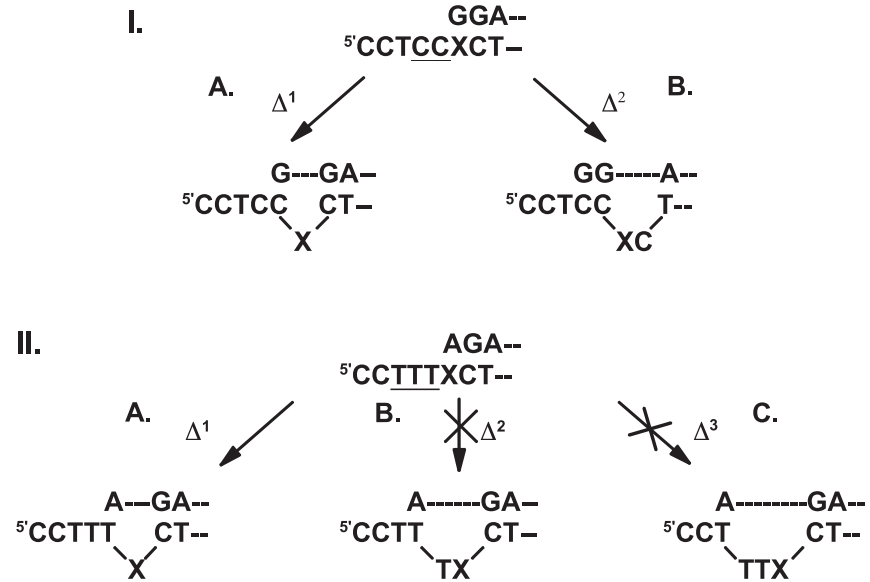

Fig. 5 Possible mechanisms of deletions on template containing iterated sequence 5 ' to the dG-AAF. (I) dG-AAF-modified 18-mer templates containing a $\mathrm{CC}$ sequence 5 ' to the lesion. (II) dG-AAFmodified 18-mer templates containing an AAA sequence 5' to the lesion.

Table 1 Frequency of nucleotide insertion and chain extension catalyzed by exo ${ }^{-}$Klenow fragment of DNA pol I

\begin{tabular}{|c|c|c|c|}
\hline & $\begin{array}{l}\text { INSERTION } \\
\begin{array}{l}\text { dNTP } \\
\downarrow \text { A - - 12-mer }\end{array}\end{array}$ & $\begin{array}{c}\text { EXTENSION } \\
\text { dTTP }\end{array}$ & -mer \\
\hline & ${ }^{5}$ CACTAXT- - & ${ }^{5}$ CACTAXT- & \\
\hline $\mathrm{N}: \mathrm{X}$ & $F_{\text {ins }}$ & $F_{\text {ext }}$ & $F_{\text {ins }} \times F_{\text {ext }}$ \\
\hline $\mathrm{C}: \mathrm{G}$ & 1.0 & 1.0 & 1.0 \\
\hline $\mathrm{C}: \mathrm{G}^{\mathrm{AAF}}$ & $6.56 \times 10^{-3}$ & $4.50 \times 10^{-6}$ & $2.95 \times 10^{-8}$ \\
\hline $\mathrm{A}: \mathrm{G}^{\mathrm{AAF}}$ & $3.78 \times 10^{-4}$ & $1.16 \times 10^{-5}$ & $5.90 \times 10^{-9}$ \\
\hline $\mathrm{G}: \mathrm{G}^{\mathrm{AAF}}$ & $1.35 \times 10^{-5}$ & $5.77 \times 10^{-6}$ & $7.79 \times 10^{-10}$ \\
\hline $\mathrm{T}: \mathrm{G}^{\mathrm{AAF}}$ & $3.79 \times 10^{-6}$ & N.D. & N.D. \\
\hline $\mathrm{C}: \mathrm{G}^{\mathrm{AF}}$ & 0.36 & $7.08 \times 10^{-2}$ & $2.54 \times 10^{-2}$ \\
\hline $\mathrm{A}: \mathrm{G}^{\mathrm{AF}}$ & $1.13 \times 10^{-5}$ & $3.98 \times 10^{-6}$ & $4.50 \times 10^{-11}$ \\
\hline $\mathrm{G}: \mathrm{G}^{\mathrm{AF}}$ & $1.10 \times 10^{-4}$ & $2.23 \times 10^{-5}$ & $2.45 \times 10^{-9}$ \\
\hline $\mathrm{T}: \mathrm{G}^{\mathrm{AF}}$ & $1.24 \times 10^{-3}$ & $5.63 \times 10^{-5}$ & $6.98 \times 10^{-8}$ \\
\hline
\end{tabular}

Frequencies of nucleotide insertion $\left(F_{\text {ins }}\right)$ and chain extension $\left(F_{\text {ext }}\right)$ were estimated by the equation: $F=\left(V_{\text {max }} / K_{\mathrm{m}}\right)_{[\text {wrong pair }]} /$ $\left(V_{\max } / K_{\mathrm{m}}\right)_{[\text {correct pair }=\mathrm{dC}: \mathrm{dG}]} . \mathrm{X}=\mathrm{dG}, \mathrm{dG}-\mathrm{AAF}$ or dG-AF.

primers (10-mer) were extended two bases prior to the lesion on template oligomers (Fig. 2) (Shibutani and Grollman, 1993). Based on the steady-state kinetic study for base insertion opposite dG-AAF, dCMP inserted opposite the lesion has the straight tendency to form deletions when the complementary base (s) is 5 ' to the lesion. In fact, the frequency of one-base deletion was increased by the following 5' sequence; G $>\mathrm{T}>\mathrm{C}>\mathrm{A}$ (Table 2). Apparently, most frequently inserted dCMP opposite the lesion pairs with G 5 ' flanking base to form a large number of one-base deletions $(12.4 \%)$. The second frequently inserted dAMP could pair with T 5 ' flanking base to form $3.7 \%$ one-base deletions.
Table 2 Effect of neighboring sequence context to the dG-AAF on the frequency of deletions

\begin{tabular}{|c|c|c|}
\hline & $\begin{array}{l}\Delta^{1} \\
(\%)\end{array}$ & $\begin{array}{l}\Delta^{2} \\
(\%)\end{array}$ \\
\hline \multicolumn{3}{|c|}{$\begin{array}{l}{ }^{5} \mathrm{CCNNNG}^{\mathrm{AAF}} \text { NNAC- } \\
\text { exo- } \\
\text { (A) }\end{array}$} \\
\hline $\mathrm{TTGG}^{\mathrm{AAF}} \mathrm{CT}$ & 12.4 & - \\
\hline $\mathrm{TTTG}^{\mathrm{AAF}} \mathrm{CT}$ & 3.7 & - \\
\hline $\mathrm{TTCG}^{\mathrm{AAF}} \mathrm{CT}$ & 0.3 & 6.8 \\
\hline $\mathrm{TTAG}^{\mathrm{AAF}} \mathrm{CT}$ & - & - \\
\hline \multicolumn{3}{|l|}{ (B) } \\
\hline $\mathrm{TGCG}^{\mathrm{AAF}} \mathrm{CT}$ & - & 40 \\
\hline $\mathrm{TTCG}^{\mathrm{AAF}} \mathrm{CT}$ & 0.3 & 6.8 \\
\hline $\mathrm{TCCG}^{\mathrm{AAF}} \mathrm{CT}$ & - & - \\
\hline $\mathrm{TACG}^{\mathrm{AAF}} \mathrm{CT}$ & - & - \\
\hline \multicolumn{3}{|l|}{ (C) } \\
\hline $\mathrm{TGCG}^{\mathrm{AAF}} \mathrm{AT}$ & - & - \\
\hline $\mathrm{TGCG}^{\mathrm{AAF}} \mathrm{TT}$ & - & - \\
\hline \multicolumn{3}{|l|}{ (D) } \\
\hline $\mathrm{GGCG}^{\mathrm{AAF}} \mathrm{CC}$ & - & 87 \\
\hline \multirow{2}{*}{\multicolumn{3}{|c|}{$\begin{array}{l}\mathrm{exo}^{+} \\
(\mathrm{E})\end{array}$}} \\
\hline & & \\
\hline $\mathrm{TGCG}^{\mathrm{AAF}} \mathrm{CT}$ & - & 0.8 \\
\hline $\mathrm{GGCG}^{\mathrm{AAF}} \mathrm{CC}$ & - & 17 \\
\hline
\end{tabular}

Using 18-mer templates containing a single dG-AAF $\left({ }^{5} \mathrm{CCNNNG}^{\mathrm{AAF}}\right.$ NNACTTTCCTCT, where $\mathrm{N}$ is $\mathrm{C}, \mathrm{A}, \mathrm{G}$ or $\mathrm{T}$ ) primed with a ${ }^{32} \mathrm{P}$ labeled 10-mer, primer extension reactions were conducted in reaction mixture containing four dNTPs and either exo ${ }^{-}$or exo $^{+}$Klenow fragment (Shibutani and Grollman, 1993).--, not detectable.

A similar phenomenon was observed in forming twobase deletions. When the base located two positions 5 ' to the lesion was changed from $\mathrm{T}$ to $\mathrm{G}$, the frequency of twobase deletion increased from $6.8 \%$ to $40 \%$. However, when $\mathrm{C}$ or $\mathrm{A}$ was located at the position, two-base deletions were not formed (Table 2). The frequencies of oneand two-base deletions also paralleled to that of base 
A.

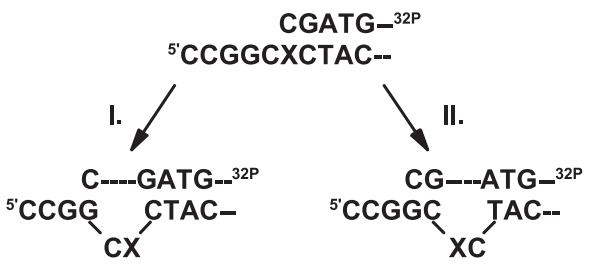

B.

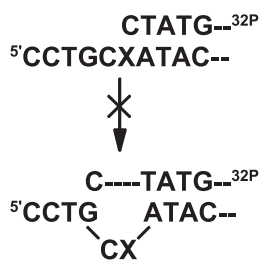

Fig. 6 Possible mechanisms of deletions on template containing a different base 3 ' to the dG-AAF

Table 3 The miscoding occurring at AAF-derived adducts during DNA synthesis catalyzed by exo ${ }^{-}$and exo $^{+}$ Klenow fragment of Pol I

\begin{tabular}{lcccc}
\hline & $\mathrm{C}$ & $\mathrm{A}$ & $\Delta^{1}$ & $\Delta^{2}$ \\
& $(\%)$ & $(\%)$ & $(\%)$ & $(\%)$ \\
exo & & & & - \\
dG & 94.0 & - & - & - \\
dG-C8-AAF & 1.4 & 0.33 & 0.3 & 6.8 \\
dG-C8-AF & 93.7 & - & - & - \\
& & & & \\
exo $^{+}$ & & & & - \\
dG & 88.1 & - & - & - \\
dG-C8-AAF & 0.4 & - & 0.1 & - \\
dG-C8-AF & 88.3 & - & - & - \\
\hline
\end{tabular}

Using a 18-mer template containing a single dG or AAFderived adduct $\left({ }^{5}\right.$ CCTTCXCTACTTTCCTCT, where $\mathrm{X}$ is $\mathrm{dG}$ or AAF-derived adduct) primed with a ${ }^{32} \mathrm{P}$-labeled 10mer, primer extension reactions were conducted in reaction mixture containing four dNTPs and either exo ${ }^{-}$or exo $^{+}$ Klenow fragment (Shibutani and Grollman, 1993). - , not detectable.

incorporation opposite dG-AAF (Shibutani and Grollman, 1993). The insertion frequency of dNTP opposite the lesion appears to be a determining factor in generating frameshift deletions.

$\mathrm{dG}-\mathrm{AF}$, a deacetylated form of dG-AAF, permitted the exclusive incorporation of dCMP, the correct base, opposite the lesion in reactions catalyzed by exo ${ }^{-}$Klenow fragment (Table 3 ). Supporting this result, $F_{\text {ins }}$ for dCMP opposite the dG-AF was much higher that that for other nucleotides and $36 \%$ of that detected for unmodified $\mathrm{dG}$ (Table 1). The rate of chain extension from the 3'-primer terminus $\left(F_{\text {ext }}\right)$ for dC:dG-AF pair was only 5 times lower than that of the $F_{\text {ins. }}$. The relative efficiency of translesion synthesis $\left(F_{\text {ins }} \times F_{\text {ext }}\right)$ past dG-AF was at least five orders of magnitude higher than that for dG-AAF when dCMP, the non-miscoding base, was incorporated opposite these lesions. Therefore, no deletions were observed during DNA synthesis past dG-AF (Shibutani and Grollman, 1993). However, $F_{\text {ext }}$ for the dC:dG-AAF pair was three orders of magnitude lower than that for the $F_{\text {ins }}$, suggesting that the chain extension reaction was blocked. If chain extension is temporarily delayed, the base inserted at the
3' primer terminus may pair to the base $5^{6}$ to the lesion, forming a misaligned intermediate that generates frameshift deletions.

\section{In Vitro Demonstration of a Mutational Hot-Spot}

A sequence containing ${ }^{5}-\mathrm{GG}^{\mathrm{AAF}}$ or ${ }^{5^{\prime}}-\mathrm{GCG}^{\mathrm{AAF}} \mathrm{C}$ produced the largest amount of one- and two-base deletions, respectively. Two-base deletions could be formed on the ${ }^{5}$ $\mathrm{GCG}^{\mathrm{AAF}} \mathrm{C}$ sequence in two pathways: dCMP located at the 3' primer terminus might pair with $\mathrm{G}$ two positions 5' to the lesion (Fig. 6AI) or CG located at the 3' primer terminus might pair with GC 5 ' to the lesion (Fig. 6AII). However, when the 3 ' flanking base was changed from $\mathrm{C}$ to $\mathrm{A}$ or $\mathrm{T}$, two-base deletions were not detected (Fig. 6B and Table 2). This indicates that the later mechanism predominates to form the two-base deletions. The 3' flanking base also influences the formation of deletions.

When sequence ${ }^{5}$ - $\mathrm{TGCG}^{\mathrm{AAF}} \mathrm{CT}$ was replaced by ${ }^{5}$ $\mathrm{GGCG}^{\mathrm{AAF}} \mathrm{CC}$, the frequency of two-base deletions increased from $40 \%$ to $87 \%$ (Table 2). ${ }^{5}-\mathrm{GGCG}^{\mathrm{AAF}} \mathrm{CC}$ is a $\mathrm{Nar}$ I restriction enzyme site sequence that is known to be a mutational hot-spot producing two-base deletions in $E$. coli (Burnouf et al., 1989; Tan et al., 2002). Mutational hot-spots observed in vivo could be demonstrated using in vitro experimental system.

\section{Effect of Proofreading on Formation of Deletions}

When the exo ${ }^{-}$Klenow fragment was used, a large amount of deletions $(7.1 \%)$ were formed from the dNMP positioned opposite dG-AAF. However, with the exo $^{+}$Klenow fragment, formation of deletions was $0.1 \%$ (Table 3). Since the dNMP positioned opposite the lesion cannot pair tightly with the bulky dG-AAF lesion, the 3 ' $\rightarrow$ 5 ' exonuclease function of this enzyme may cleave dNMP from the 3' primer terminus, resulting in a limited formation of deletions. The proofreading may act to minimize deletions. Interestingly, the frequency of two-base deletions generated by ${ }^{5}-\mathrm{GGCG}^{\mathrm{AAF}} \mathrm{CC}$ was $17 \%$. The mutational hot-spot sequence such as the Nar I site may be resistant to the proofreading, resulting in a high frequency of deletions. 


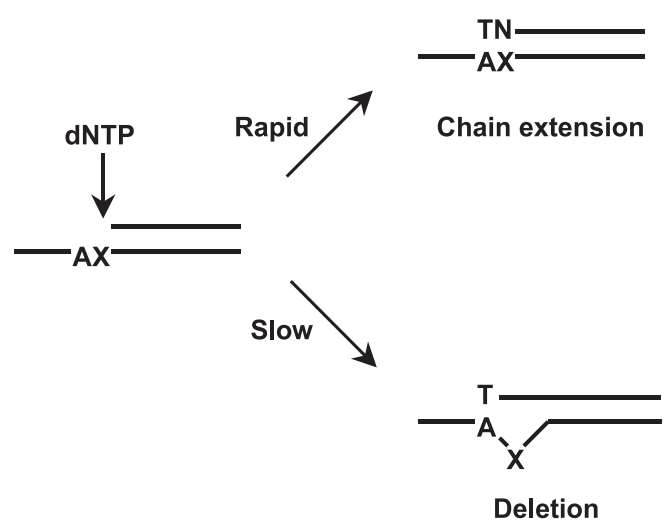

Fig. 7 Mechanism for frameshift (deletion) mutation

\section{Frequency of Deletions Generated on Templates Containing Iterated Sequences 5' to dG-AAF Lesion}

Slipped mispairing was proposed to account for the frameshift observed in iterated nucleotide sequences (Streisinger et al., 1966; 1985). Templates containing an iterated nucleotide sequence 5 ' to the dG-AAF were used to investigate the formation of frameshift deletions. When TTT was 5' to dG-AAF in the template, deletions could form in three pathways: dAMP located at the 3' primer terminus might pair with $\mathrm{T} 5$ ' flanking to form one-base deletions (Fig. 5IIA), with T two positions 5' to the lesion to form two-base deletions (Fig. 5IIB), or with T three positions 5 ' to the lesion to form three-base deletions (Fig. 5IIC). When a shorter primer (10-mer) was used to initiate the primer extension reaction from two bases prior to the lesion, only one-base deletions were generated during DNA synthesis (Table 2) (Shibutani and Grollman, 1993), indicating that the first pathway was thought to be operative. This result was consistent with data obtained using the 13-mer with dA (Shibutani et al., 2004). Similar results were observed when GG or AA was 5 ' to the dGAAF. Thus, formation of the shorter deletion predominated.

\section{Formation of Deletions Induced by SOS- Inducible DNA Polymerases}

A number of novel $E$. coli DNA polymerases, including pol IV and pol V, have recently been characterized (reviewed by Goodman, 2002). These enzymes lack 3' $\rightarrow$ 5 ' proofreading exonuclease activity and exhibit high error rates even on non-damaged DNA templates. Pol IV and $\mathrm{V}$, in addition to pol II, are the SOS-inducible DNA polymerases that may generate mutation during translation synthesis past various forms of damaged DNA (Napolitano et al., 2000; Wagner et al., 2002). Using mutated cells lacking specific polymerase (s), pol II and pol $\mathrm{V}$ were found to be primarily involved in the formation of two-base deletions on GC repetitive sequences contain- ing a single dG-AAF (Napolitano, et al., 2000; Wagner et al., 2002). In experiments with pol IV, one- and two-base deletions were formed at the dG-AAF lesion (Suzuki et al., 2001), following the mechanism proposed for pol I (Shibutani and Grollman, 1993). However, translesion synthesis was strongly blocked at the lesion site; deletions were formed only when large amounts of this enzyme were used (Suzuki et al., 2001). Pol IV is unlikely to be involved in translesion synthesis past dG-AAF, as reported earlier by Napolitano et al. (2000).

\section{Deletions Generated by Mammalian DNA Polymerases}

DNA pol $\alpha$, a replicative enzyme in mammalian cells, promoted deletions on site-specifically modified template with an apurinic/apyridiminic site (Shibutani et al., 1997) or 3, $N^{4}$-etheno-dC (Shibutani et al., 1996). Human DNA pol $\kappa$, a homolog of the E. coli DinB polymerase (pol IV) (Gerlach et al., 1999; Ogi et al., 1999) , also generated oneand two-base deletions during DNA synthesis past dGAAF (Suzuki et al., 2001) or estrogen-derived DNA adduct (Suzuki et al., 2004). The formation of deletions induced by pol $\alpha$ and pol $\kappa$ was consistent with our proposed mechanism of deletions established using the $E$. coli enzyme (Shibutani and Grollman, 1993). Thus, similar mechanisms of frameshift deletion appear to apply to mammalian cells.

When a dG- $N^{2}$-tamoxifen-modified template $\left({ }^{5} \mathrm{CAT}\right.$ GCTGATGAATTCCTTCXCTTCTTTCCTCTCCCTTT, where $\mathrm{x}$ is $\mathrm{dG}-\mathrm{N}^{2}$-tamoxifen lesion) primed with a 10-mer $\left({ }^{5}\right.$ AGAGGAAAGA) was used, large amounts of five-base deletions were formed during translesion synthesis catalyzed by mammalian pol $\alpha$ or pol $\beta$ (Shibutani and Dasaradhi, 1997). In this case, dGMP was preferentially inserted opposite the lesion. The newly inserted dGMP opposite the lesion and its 5' bases, GAAG, of the primer could be paired with CCTTC 5' to the lesion, resulting in the formation of five-base deletions. This indicates that the formation of deletions is also dependent on the frequency of dNTP insertion opposite the lesion. Our proposed mechanism of deletion (Shibutani and Grollman, 1993) could be used to predict "long" ( $>3$ bases) deletions.

\section{Conclusion}

As shown in Fig. 7, base substitutions predominate when chain extension from the 3' terminus rapidly follows insertion of an "incorrect" base opposite the lesion. However, if chain extension is temporarily delayed, the nucleotide inserted at the 3 ' primer terminus may pair to the base 5 ' to the lesion, forming a misaligned intermediate that generates one- and/or longer deletions. Thus, frameshift deletions are primarily generated following a mechanism; namely, misinsertion preceding misalign- 
ment. The ability to generate deletions depends primarily on three factors: $(a)$ the nature of the base inserted opposite the lesion, (b) sequence context to the lesion, and $(c)$ the overall rate of translesion DNA synthesis past the lesion. This mechanism may be applied to predict the nature of deletions generated by a variety of DNA adducts and by prokaryotic and eukaryotic DNA polymerases.

\section{Acknowlegements}

This research was supported by Grant ES04068 from the National Institutes of Environmental Health Sciences.

\section{References}

Bebenek, K. and T.A. Kunkel (1990) Frameshift errors initiated by nucleotide misincorporation, Proc. Natl. Acad. Sci. USA, 87, 49464950.

Burnouf, D., P. Koehl and R.P.P. Fuchs (1989) Single adduct mutagenesis: strong effect of the position of a single acetylaminofluorene adduct within a mutation hot spot, Proc. Natl. Acad. Sci. USA, 86, 4147-4151.

Fowler, R.G., G.E. Degnen and E.C. Cox (1974) Mutational specificity of a conditional Escherichia coli mutator, mutD5, Mol. Gen. Genet., 133, 179-191.

Fresco, J.R. and B.M. Alberts (1960) The accommodation of noncomplementary bases in helical polyribonucleotides and deoxyribonucleic acids, Proc. Natl. Acad. Sci. USA, 46, 311-321.

Gerlach, V.L., L. Aravind, G. Gotway, R.A. Schultz, E.V. Koonin and E.C. Friedberg (1999) Human and mouse homologs of Escherichia coli DinB (DNA polymerase IV), members of the UmuC/DinB superfamily, Proc. Natl. Acad. Sci. USA, 96, 1192211927.

Goodman, M.F. (2002) Error-prone repair DNA polymerases in prokaryotes and eukaryotes, Annu. Rev. Biochem., 71, 17-50.

Kunkel, T.A. (1990) Misalignment-mediated DNA synthesis errors, Biochemistry, 29, 8003-8011.

Kunkel, T.A. and A. Soni (1988) Mutagenesis by transient misalignment, J. Biol. Chem., 263, 14784-14789.

Mendelman, L.V., M.S. Boosalis, J. Petruska and M.F. Goodman (1989) Nearest neighbor influences on DNA polymerase insertion fidelity, J. Biol. Chem., 264, 14415-14423.

Mendelman, L.V., J. Petruska and M.F. Goodman (1990) Base mispair extension kinetics, J. Biol. Chem., 265, 2338-2346.

Napolitano, R., R. Janel-Bintz, J. Wagner and R.P.P. Fuchs (2000) All three SOS-inducible DNA polymerases (Pol II, Pol IV and Pol V) are involved in induced mutagenesis, EMBO J., 19, 6259-6265.

Ogi, T., T. Kato Jr., T. Kato and H. Ohmori (1999) Mutation enhancement by DINB1, a mammalian homologue of the Escherichia coli mutagenesis protein dinB, Genes Cells, 4, 607-618.

Ripley, L.S. (1990) Frameshift mutation: determinations of specificity, Annu. Rev. Genet., 24, 189-213.

Shibutani, S. (1993) Quantitation of base substitutions and deletions induced by chemical mutagens during DNA synthesis in vitro, Chem. Res. Toxicol., 6, 625-629.

Shibutani, S. and A.P. Grollman (1993) On the mechanism of frameshift (deletion) mutagenesis in vitro, J. Biol. Chem., 268, 11703-11710

Shibutani, S., N. Suzuki, Y. Matsumoto and A.P. Grollman (1996) Miscoding properties of $3, N^{4}$-etheno-2'-deoxycytidine in reactions catalyzed by mammalian DNA polymerases, Biochemistry, 35 , 14992-14998.

Shibutani, S. and L. Dasaradhi (1997) Miscoding potential of tamoxifen-derived DNA adducts: $\alpha$ - $\left(N^{2}\right.$-deoxyguanosinyl $)$ tamoxifen, Biochemistry, 36, 13010-13017.

Shibutani, S., M. Takeshita and A.P. Grollman (1997) Translesional synthesis on DNA templates containing a single abasic site. A mechanistic study of the "A rule”, J. Biol. Chem., 272, 1391613922.

Shibutani, S., N. Suzuki and A.P. Grollman (2004) Mechanism of frameshift (deletion) mutagenesis generated by an acetylaminofluorene-derived DNA adduct in vitro, Under reviewed by Biochemistry.

Streisinger, G., Y. Okada, J. Emrich, J. Newton, A. Tsugita, E. Terzaghi and M. Inouye (1966) Frameshift mutations and the genetic code, Cold Spring Harbor Symp. Quant. Biol., 31, 77-84.

Streisinger, G. and J.E. Owen (1985) Mechanisms of spontaneous and induced frameshift mutation in Bacteriophage T4, Genetics, 109, 633-659.

Suzuki, N., S. Itoh, K. Poon, C. Masutani, F. Hanaoka, H. Ohmori, I. Yoshizawa and S. Shibutani (2004) Translesion synthesis past estrogen-derived DNA adducts by human DNA polymerases $\eta$ and $\kappa$, Biochemistry, 43, 6304-6311.

Suzuki, N., E. Ohashi, K. Hayashi, H. Ohmori, A.P. Grollman and S. Shibutani (2001) Translesional synthesis past acetylaminofluorene-derived DNA adducts catalyzed by human DNA polymerase $\kappa$ and Escherichia coli DNA polymerase IV, Biochemistry, 40, 15176-15183.

Tan, X., N. Suzuki, A.P. Grollman and S. Shibutani (2002) Mutagenic events in Escherichia coli and mammalian cells generated in response to acetylaminofluorene-derived DNA adducts positioned in the Nar I restriction enzyme site, Biochemistry, 41, 14255-14262.

Wagner, J., H. Etienne, R. Janel-Bintz and R.P. Fuchs (2002) Genetics of mutagenesis in E. coli: various combinations of translesion polymerases ( pol II, IV and V) deal with lesion/sequence context diversity, DNA Repair, 1, 159-167. 